\title{
Strong and Electromagnetic Interactions at SPS Energies
}

\section{Andrzej RYBICKI ${ }^{* \dagger}$}

H. Niewodniczański Institute of Nuclear Physics, Polish Academy of Sciences

E-mail: andrzej.rybickidcern.ch

Particle production in peripheral $\mathrm{Pb}+\mathrm{Pb}$ collisions has been measured at a beam energy of 158 $\mathrm{GeV}$ per nucleon, corresponding to $\sqrt{s_{N N}} \approx 17.3 \mathrm{GeV}$. The measurements provide full double differential coverage in a wide range of longitudinal and transverse momenta, including the central ("mid-rapidity") area and extending far into the projectile fragmentation region. The resulting analysis shows the heavy ion reaction as a mixture of different processes. In particular, surprising phenomena, like the presence of large and strongly varying structures in the shape of the double differential cross section $d^{2} \sigma / d x_{F} d p_{T}$, are induced by the final state electromagnetic interaction between produced particles and the charged spectator system. This effect is largest at low transverse momenta, where it results in a deep valley in the $x_{F}$-dependence of the produced $\pi^{+} / \pi^{-}$ ratio. The basic characteristics of the electromagnetic phenomenon described above agree with the results of a theoretical analysis, performed by means of a simple model of the peripheral $\mathrm{Pb}+\mathrm{Pb}$ reaction. Versatile information on the heavy ion collision mechanism becomes therefore available. In particular, the electromagnetic effect is sensitive to the initial conditions of particle production (time of final state hadron emission, distance of the formation zone from the two spectator systems). As a result, it may provide new information on the space and time evolution of the particle production process.

European Physical Society Europhysics Conference on High Energy Physics

July 16-22, 2009

Krakow, Poland

\footnotetext{
*Speaker.

$\dagger$ All the experimental results and other experimental input used in this paper were obtained in the framework of the NA49 Collaboration. The theoretical calculations were performed in collaboration with A. Szczurek (H. Niewodniczański Institute of Nuclear Physics, Polish Academy of Sciences).
} 

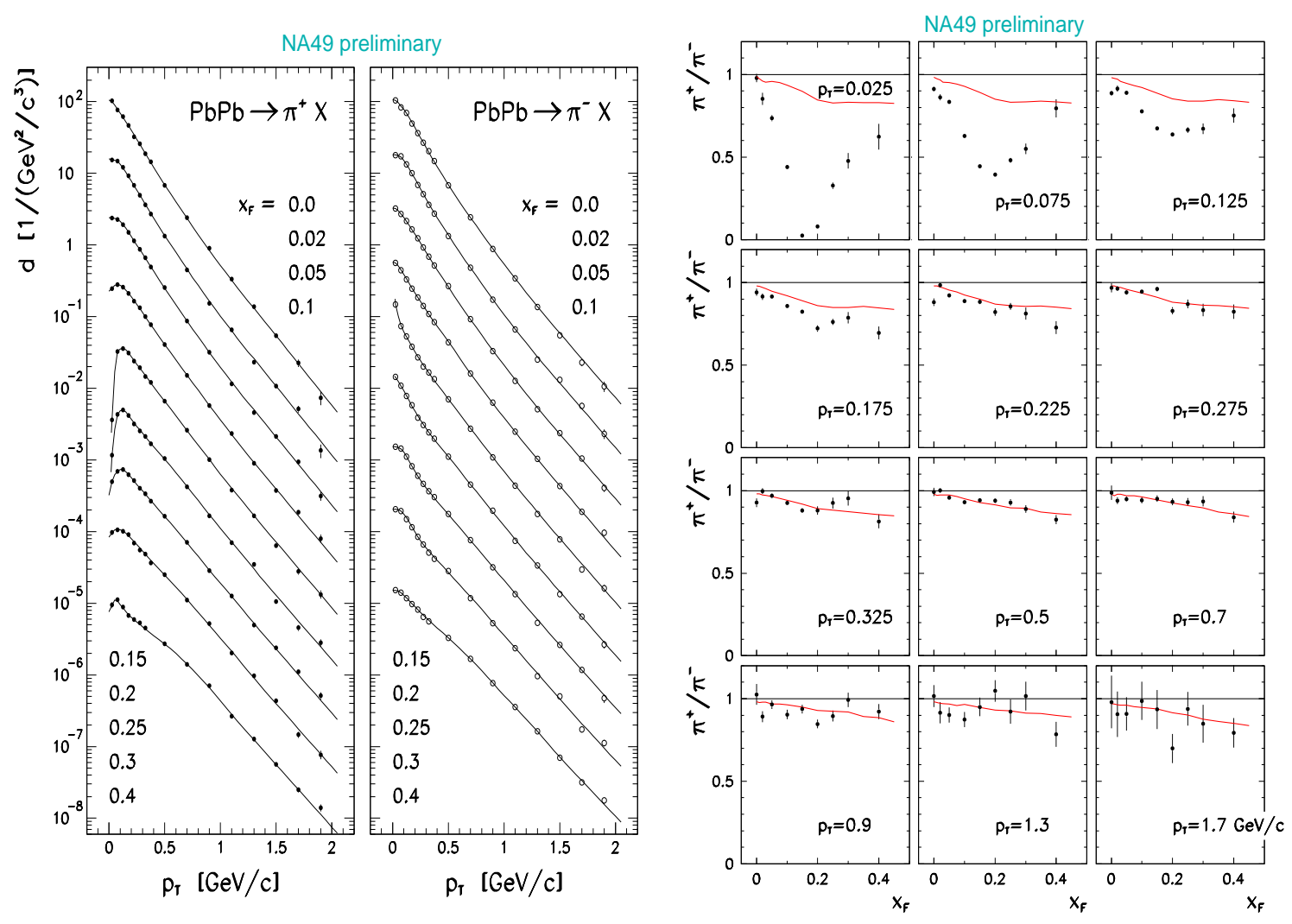

Figure 1: Left (vertical panels): invariant density $d=E \cdot d^{3} n / d p^{3}$ of positive and negative pions produced in peripheral $\mathrm{Pb}+\mathrm{Pb}$ collisions, drawn as a function of $p_{T}$ at fixed values of the Feynman variable $x_{F}=2 p_{L} / \sqrt{s}$ (nucleon-nucleon c.m.s.). Both the data points and the interpolated lines are successively multiplied by 0.2 . Right (multiplot): $x_{F}$-dependence of the charged pion ratio $\pi^{+} / \pi^{-}$in peripheral $\mathrm{Pb}+\mathrm{Pb}$ reactions (data points), compared to the same ratio for nucleon-nucleon reactions (solid line).

The NA49 experiment at the CERN SPS [1] provided an extensive set of experimental data on particle production in hadron-hadron, hadron-nucleus and nucleus-nucleus reactions. This paper focuses on phenomena occurring in peripheral $\mathrm{Pb}+\mathrm{Pb}$ collisions at a beam energy of $158 \mathrm{GeV} /$ nucleon. Preliminary results from a double differential measurement of charged pion production in these collisions are compared to nucleon-nucleon interactions. A specific electromagnetic effect observed in $\mathrm{Pb}+\mathrm{Pb}$ reactions is interpreted by means of theoretical model calculations.

The peripheral $\mathrm{Pb}+\mathrm{Pb}$ sample constitutes a part of a "minimum bias" data set. It is isolated by a multiplicity cut corresponding to 150-300 charged particles seen in the detector, to be compared to up to 1500 tracks for most central $\mathrm{Pb}+\mathrm{Pb}$ collisions. The measurement of $\pi^{+}$and $\pi^{-}$production is presented in Fig. 1 (left panels). It starts at $x_{F}=0$ which corresponds to mid-rapidity and extends up to $x_{F}=0.4$ which is, for pions at low transverse momentum, well above beam rapidity.

The ratios of charged pions produced in the $\mathrm{Pb}+\mathrm{Pb}$ reaction, $\pi^{+} / \pi^{-}$, are shown in Fig. 1 (right panels). These are compared to the same ratios for pions produced in nucleon-nucleon reactions ${ }^{1}$. At higher transverse momenta there is an overall agreement between the two reaction types. Be-

\footnotetext{
1 "Nucleon-nucleon" means a mixture of $40 \%$ colliding protons and $60 \%$ neutrons, matching the isospin content of the $\mathrm{Pb}$ nucleus. The resulting $\pi^{+} / \pi^{-}$ratios are deduced from NA49 $\mathrm{p}+\mathrm{p}$ data [2] on the basis of isospin symmetry [3].
} 

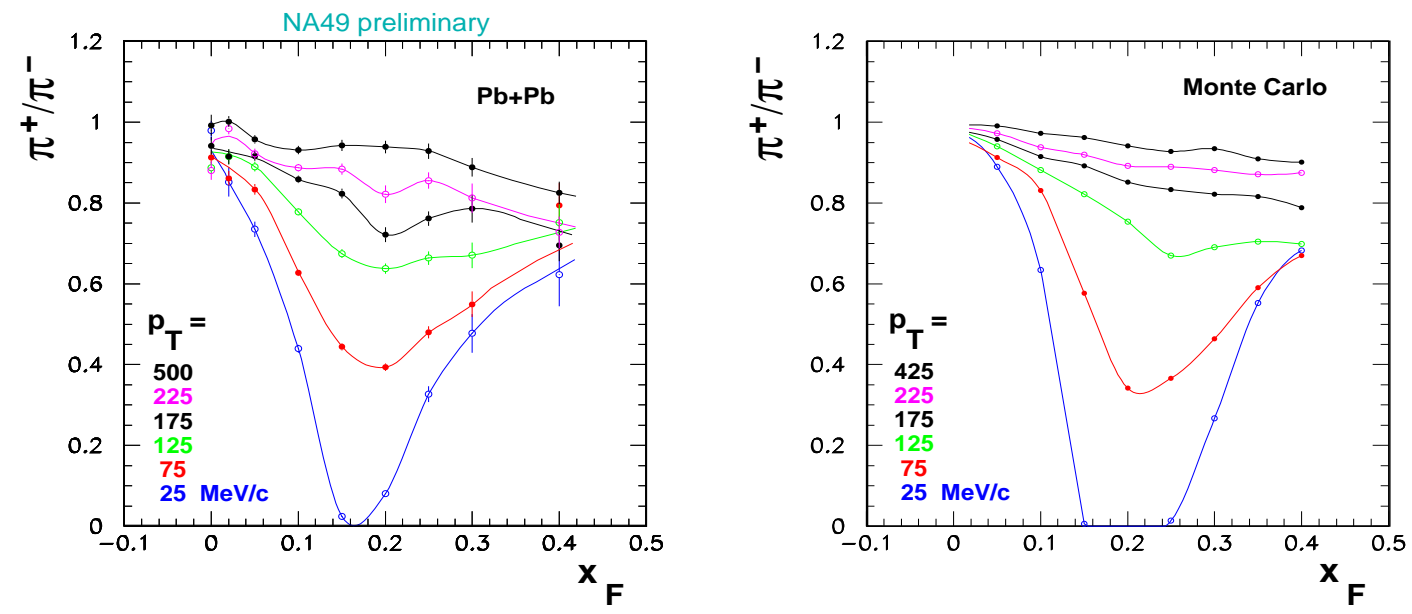

Figure 2: Left: $\pi^{+} / \pi^{-}$ratios in peripheral $\mathrm{Pb}+\mathrm{Pb}$ collisions, drawn as a function of $x_{F}$ at several values of $p_{T}$ (listed from top to bottom curve). Right: $\pi^{+} / \pi^{-}$ratios obtained by Monte Carlo simulation.

low $p_{T} \approx 0.2 \mathrm{GeV} / \mathrm{c}$, however, an increasingly violent drop of $\pi^{+} / \pi^{-}$can be observed in $\mathrm{Pb}+\mathrm{Pb}$ collisions when approaching $p_{T}=0$. This is shown in more detail in Fig. 2 (left panel). The $\pi^{+} / \pi^{-}$ratio in $\mathrm{Pb}+\mathrm{Pb}$ reactions displays a smooth two-dimensional structure, with a characteristic steep valley approaching zero at low $p_{T}$. Such low values of the $\pi^{+} / \pi^{-}$ratio suggest that this phenomenon is caused by the electromagnetic interaction which is not constrained by isospin symmetry. As the effect is strongest close to $x_{F}=0.15 \approx \frac{m_{\pi}}{m_{N}}$ which corresponds to pions moving longitudinally at spectator velocities, its origin appears to be the Coulomb repulsion (attraction) of positive (negative) pions by the highly charged spectator system emerging from the peripheral collision. This is confirmed by an electromagnetic Monte Carlo simulation, Fig. 2 (right panel), which properly describes the basic characteristics of the data.

A theoretical study of this effect was performed by means of a simplified model: the two outgoing spectator systems were imagined as two Lorentz-contracted spheres, while final state pion emission took place from the interaction point after a given emission time $t_{E}$ (more details in [4]). One result is shown in Fig. 目 (left panels). The two-dimensional structure from Fig. 目 appears now as a part of a broader distortion pattern. The shape of the two valleys induced by the two spectator systems, and the electromagnetic modification of $\pi^{+} / \pi^{-}$ratios at higher $\left|x_{F}\right|$, both depend on initial conditions imposed by the emission time $t_{E}$. As in the considered model, $t_{E}$ is equivalent to the distance between the pion formation zone and the spectator systems, it can be altogether concluded that the spectator-induced electromagnetic distortion of pion spectra carries new information on the space-time evolution of the non-perturbative process of particle production.

A first attempt at an extension of the above study into the domain of strangeness production is shown in Fig. 3 (right panels). The increase of the final state particle mass from pion to kaon has definite kinematic consequences for the electromagnetic effect ${ }^{2}$. Compared to these shown for pions, the two valleys in $K^{+} / K^{-}$ratios are displaced towards much higher values of $\left|x_{F}\right|$. They are also broader, both in $x_{F}$ and in $p_{T}$. While the overall distortion pattern clearly remains sensitive

\footnotetext{
${ }^{2}$ This introductory study focuses on the purely kinematic aspects of the electromagnetic effect for kaons. A more detailed analysis, including a more realistic description of $\mathrm{K}^{+}$and $\mathrm{K}^{-}$production yields and spectra, is in progress.
} 


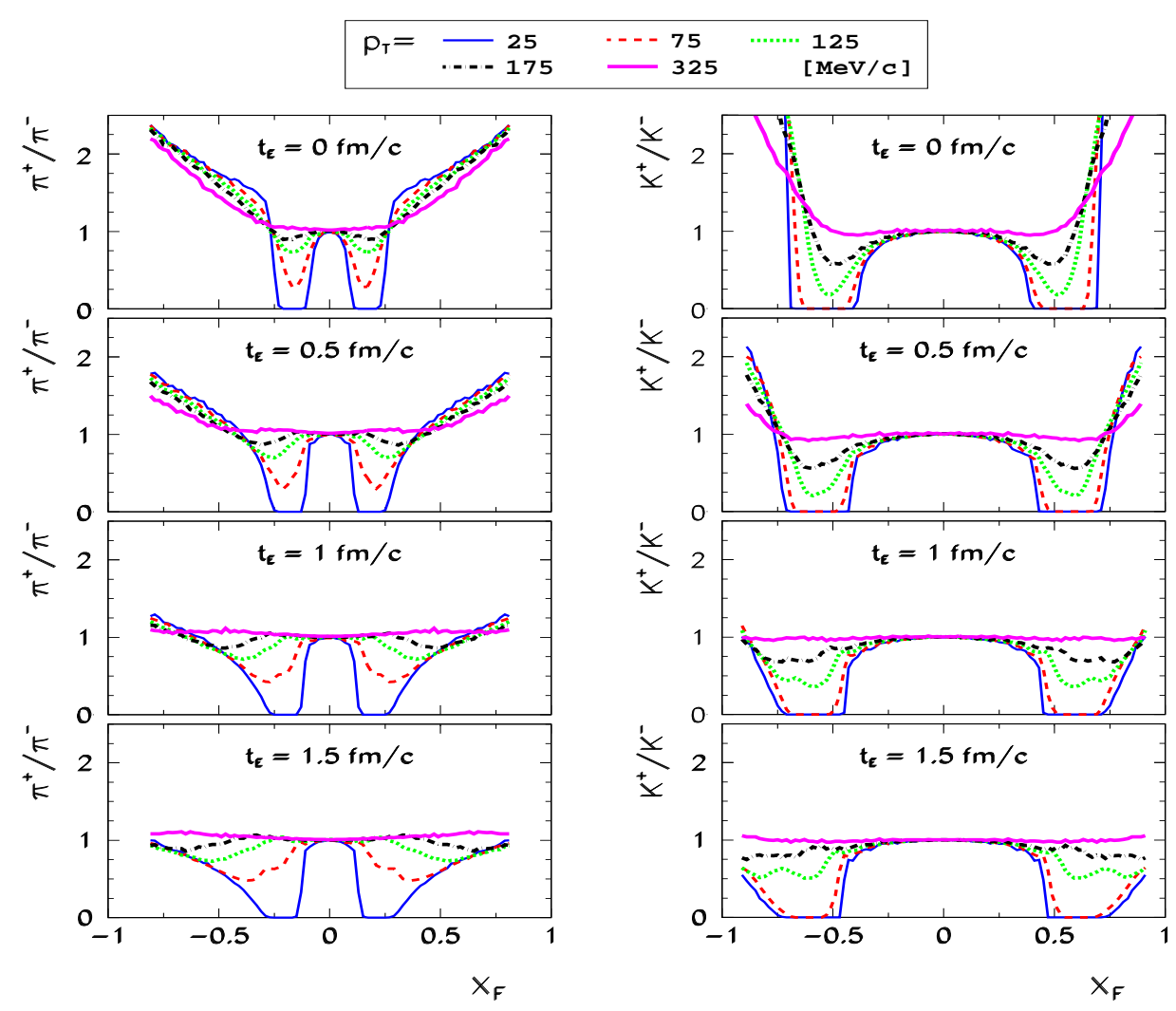

Figure 3: Dependence of the electromagnetic effect on initial conditions for pions (left) and kaons (right). The $x_{F}$-dependence of the ratio $\pi^{+} / \pi^{-}$(left) and $K^{+} / K^{-}$(right) at several values of $p_{T}$ is shown for different assumed values of the emission time $t_{E}$. The left panels are re-drawn from [

to initial conditions imposed on kaon production, the region of highest sensitivity is shifted in the direction of very high $\left|x_{F}\right|$.

\section{Acknowledgments}

This work was supported by the Polish Ministry of Science and Higher Education under grant no. N N202 078735.

\section{References}

[1] NA49 Collab., S. Afanasev et al., The NA49 large acceptance hadron detector, Nucl. Instrum. Meth. A 430 (1999) 210.

[2] NA49 Collab., C. Alt et al., Inclusive production of charged pions in $p+p$ collisions at 158-GeV/c beam momentum, Eur. Phys. J. C 45 (2006) 343 [hep-ex/ 051000 9].

[3] O. Chvala (NA49 Collab.), On the importance of isospin effects for the interpretation of nuclear collisions, Eur. Phys. J. C 33 (2004) S615 [hep-ex/ 0405053 ].

[4] A. Rybicki, A. Szczurek, Spectator electromagnetic effect on charged pion spectra in peripheral ultrarelativistic heavy ion collisions, Phys. Rev. C 75 (2007) 054903 [nucl-th/ 0610036 ]. 